\title{
Interaction of hydrogen with dislocations in tungsten: An atomistic study
}

\author{
Petr Grigorev ${ }^{\mathrm{a}, \mathrm{b}}$, Dmitry Terentyev ${ }^{\mathrm{a}}$, Giovanni Bonny ${ }^{\mathrm{a}}$, Evgeny E. Zhurkin ${ }^{\mathrm{c}}$, Guido Van Oost ${ }^{\mathrm{b}}$, \\ Jean-Marie Noterdaeme ${ }^{b}$ \\ ${ }^{a}$ SCK $\bullet$ CEN, Nuclear Materials Science Institute, Boeretang 200, Mol, 2400, Belgium \\ ${ }^{b}$ Ghent University, Applied Physics EA17 FUSION-DC, St.Pietersnieuwstraat, 41 B4 B-9000, Gent, \\ Belgium \\ ${ }^{c}$ Department of Experimental Nuclear Physics K-89, Institute of Physics, nanotechnology and \\ telecommunications, St.Petersburg State Polytechnical University, 29 Polytekhnicheskaya str., 195251, \\ St.Petersburg, Russia
}

\begin{abstract}
The interaction of interstitial hydrogen with a dislocation and point defects in tungsten is studied by means of atomistic simulations. Two different types of interatomic potentials were tested by comparing their results with available ab initio data. The recently developed embedded atom method potential showed a better agreement with ab initio results than the bond order potential. Static calculations involving screw and edge dislocations showed that hydrogen is attracted to the dislocation core in both cases. It is also found that hydrogen atoms prefer to arrange themselves as elongated clusters on dislocation lines. Molecular dynamics simulations of hydrogen migration along the edge dislocation core confirmed the results of the static calculations and demonstrated a strong attraction to the dislocation core and one-dimensional migration along it.
\end{abstract}

\section{Introduction}

Tungsten (W) is one of the currently considered in-vessel plasma-facing materials for ITER [1]. During ITERs exploitation, cyclic thermal stresses coupled with radiation damage and trapping of plasma components (retention) impose a serious uncertainty regarding the lifespan of the components made of $\mathrm{W}$. Hydrogen $(\mathrm{H})$ retention is a specific problem, since it has a dual impact defining the degradation of $\mathrm{W}$-based components. On the one hand, the maximum retention is limited by the safety limits, and on the other hand, the storage of hydrogen provokes further embrittlement to be added to the detrimental effect of neutron irradiation and thermal fatigue.

Despite significant efforts done in past investigations to explore the main mechanisms of $\mathrm{H}$ retention in $\mathrm{W}$ [2-6], a complete physical model capable of describing a broad set of experimental data does not yet exist. In our recent works $[7,8]$, we have drawn attention to the role played by dislocations in the trapping, transport and nucleation of hydrogen bubbles. Based on the $a b$ initio calculations we have proposed the so-called 'jog-punching' process as the mechanism to explain the transformation of a meta-stable hydrogen cluster into a stable hydrogen-vacancy cluster - nucleus for a future hydrogen bubble [7].

The idea of the jog-punching mechanism and the obtained ab initio data was then implemented in a new theoretical model for the $\mathrm{H}$ retention based on $\mathrm{H}$ trapping at dislocations and transport to the surface via the dislocation network [8]. Such a model was used to explain the experimentally observed saturation of $\mathrm{H}$ retention with dose in different $\mathrm{W}$ grades under high flux plasma implantation conditions. One of the principal conditions of this model was the assumption about transport of hydrogen atoms along a dislocation network. Although the $a b$ 
initio calculations have demonstrated that the migration barrier for $\mathrm{H}$ to move along the core of a $1 / 2<111>$ screw dislocation is smaller than the bulk migration energy, no direct dynamic simulations have so far been performed to demonstrate the preferential diffusion of $\mathrm{H}$ in the dislocation core. Moreover, ab initio techniques are not suitable for considering defects that produce large stress fields such as edge dislocations and therefore classical molecular dynamics (MD) studies are still needed to close the gap.

In this work we perform an MD study to characterize the interaction of $\mathrm{H}$ with screw and edge dislocations at zero Kelvin and finite temperature. The study is performed using two interatomic potentials, namely: the bond order type potential (BOP) developed by Li et.al. [9, 10], and the recently derived embedded atom method (EAM) potential [11]. The results are compared with available ab initio data. By comparing the performance of the two potentials regarding the description of $\mathrm{H}$-vacancy and $\mathrm{H}$-dislocation interaction, we conclude that the EAM potential provides a better agreement with $a b$ initio data and therefore will be used for further MD investigations. Based on the preliminary MD data obtained here, we conclude that $\mathrm{H}$ exhibits strong attractive interaction with the core of the $1 / 2<111>\{110\}$ edge dislocation and at finite temperature performs enhanced one-dimensional migration as compared to the bulk diffusivity.

\section{Computational details.}

As mentioned before, we used two different types of the interatomic potentials, namely: BOP and EAM. There were two different versions of the EAM potential, referred to as "EAM1" and "EAM2" in [11]. The BOP potential, developed in [9, 10], was fitted to the $\mathrm{H}$ interaction with point defects in $\mathrm{W}$. It reproduces very well $\mathrm{H}-\mathrm{W}$ molecules and geometry of $\mathrm{H}$-vacancy system (i.e. off-centered position of $\mathrm{H}$ displaced along <100> direction), however the resulting $\mathrm{H}$-vacancy binding energy slightly differs from the $a b$ initio result. Both EAM potentials were based on the interatomic potential for bcc W named "EAM2" from work [12]. The choice was made after critical review of 19 different EAM potentials given in [13]. For the EAM1 potential, emphasis was put on a quantitative reproduction of $a b$ initio data for the binding between $\mathrm{H}-\mathrm{H}$, $\mathrm{He}-\mathrm{He}$ and $\mathrm{H}-\mathrm{He}$ pairs [11]. The off-center position of a $\mathrm{H}$ atom in a vacancy as predicted by DFT [14] was not considered, and therefore both $\mathrm{H}$ and $\mathrm{He}$ are described by pair potentials only. For the EAM2 potential, the focus was made on the stabilizing $\mathrm{H}$ in an off-center position in the vacancy and therefore an embedding function was added for $\mathrm{H}$. Both types of the potentials predict the tetrahedral position for a $\mathrm{H}$ atom as the most favorable in bulk $\mathrm{W}$, which is important for this work as we focus on the calculation of the binding between $\mathrm{H}$ atoms and defects.

MS and MD calculations were performed using the LAMMPS simulation package [15], where the above-mentioned interatomic potentials were implemented. Simulations were performed in bcc W. All MD simulations were performed using a classical MD algorithm in the microcanonical NVE ensemble with a timestep of 1 fs. All MS calculations were performed using a conjugate gradient algorithm embedded in the LAMMPS package with an energy change tolerance of $10^{-10} \mathrm{eV} /$ atom.

The size of the crystallite used in simulations containing point defects (interstitial $\mathrm{H}$, vacancies and their combinations) was $10 \times 10 \times 10 \mathrm{a}_{0}{ }^{3}\left(\mathrm{a}_{0}\right.$ is the lattice constant predicted by the potential: $3.14 \AA$ for EAM potentials and $3.165 \AA$ for BOP), thus it contained 2000 atoms before any point defect or cluster was introduced. Periodic boundary conditions were applied in all three directions. For calculations involving a $1 / 2<111>$ screw dislocation box size was $152.9 \times 78.1 \times 32.6$ 
$\AA$ (25920 atoms) with axis orientations [1-10], [11-2], [111], respectively for X,Y,Z principal axes. Free surfaces along the $\mathrm{X}$ and $\mathrm{Y}$ were introduced, while periodic boundary conditions were applied along the $\mathrm{Z}$ direction, coinciding with the orientation of the dislocation line and dislocation Burgers vector. For the calculations involving a $1 / 2<111>\{110\}$ edge dislocation, the box size was $80.9 \times 38.8 \times 111.7 \AA$ (22155 atoms). The X,Y,Z axes orientations were [111], [11-2], [-110] with periodic conditions imposed along the $\mathrm{X}$ and $\mathrm{Y}$ directions and free surfaces perpendicular to the $\mathrm{Z}$ direction. The dislocation line was oriented along [11-2] direction.

Estimation of the binding energy for point defects ( $\mathrm{H}-\mathrm{H}$ pairs and $\mathrm{H}$-vacancy clusters) as well as the binding energy for $\mathrm{H}_{\mathrm{N}}$ clusters with the dislocation core requires the calculation of the total energy of the atomic system containing these defects being placed together and isolated. The corresponding binding energy of $\mathrm{H}$ with different types of lattice defects was defined as:

$$
E_{H D}^{B}=E_{H}+E_{D}-E_{H D}-N_{a t} E_{c o h}
$$

where $E_{H D}$ is the total energy of the system when $\mathrm{H}$ is attached to the defect, $E_{H}, E_{D}$ the total energy of the system containing only $\mathrm{H}$ or only a single considered lattice defect (i.e. a vacancy, another interstitial $\mathrm{H}$ atom or dislocation) correspondingly. $N_{a t} E_{c o h}$ is introduced to respect the particle number balance and to compensate for the different number of matrix $\mathrm{W}$ atoms present in the configurations corresponding to $E_{H D}, E_{H}$ and $E_{D}$ energies. Thus, $N_{a t}$ is the number of atoms, $E_{c o h}$ - is the energy per atom in pure W. In this notation, a positive value of the binding energy corresponds to attraction between the defects.

In order to estimate the diffusion parameters of $\mathrm{H}$ atoms and validate ab initio predictions regarding the preferential one dimensional $\mathrm{H}$ migration along the dislocation core, a number of MD calculations were performed at finite temperature, $T$. The main goal was to obtain the diffusion coefficient as a function of temperature, which would allow one to extract the preexponential factor $D_{0}$ and activation energy $E_{m}$ using the Arrhenius type equation:

$$
D=D_{0} \exp \left(\frac{-E_{m}}{k_{B} T}\right)
$$

In each MD run that lasted for a time $\tau$, the trajectory of the $\mathrm{H}$ atom was followed and visualized to quantify the dimensionality of the $\mathrm{H}$ motion, which depends on the ambient temperature and type of defect present in the system. Then, the mean square displacement $\overline{R^{2}}$ of the position of the $\mathrm{H}$ atom was calculated to obtain the diffusion coefficient using the wellknown Einstein equation:

$$
D_{n}(T)=\frac{\overline{R_{n}^{2}}}{2 n \tau}(T)
$$

where $n$ is dimensionality of the motion (i.e., $n=1$ for one dimensional migration along a dislocation core, and $n=3$ for three dimensional bulk diffusion), $\tau$ - simulation time.

\section{Results and discussion}

\subsection{Benchmark calculations}

Prior considering complex interactions of $\mathrm{H}_{\mathrm{N}}$ clusters with dislocations we performed a set of benchmark calculations to compare the results with well established ab initio data. The 
benchmark calculations involved the characterization of the $\mathrm{H}-\mathrm{H}$ and $\mathrm{H}$-vacancy interaction as a function of distance, as schematically shown in Fig. 1. The corresponding binding energy as a function of distance, determined by the atomic positions after complete relaxation, is given in Figs. 2 and 3 for the $\mathrm{H}-\mathrm{H}$ and $\mathrm{H}$-vacancy interaction, respectively.

Let us first consider the results for H-H interaction presented in Fig. 2. Although all the presented curves provide the same trend as the ab initio method, we can see that the repulsive interaction in the first nearest neighbor position is significantly overestimated by the BOP. For the configurations corresponding to the interaction distance of $0.62 \mathrm{a}_{0}$ and $0.7 \mathrm{a}_{0}$, the BOP potential does not return stable configuration and the $\mathrm{H}$ atoms displace either to the $0.55 \mathrm{a}_{0}$ or 0.8 $\mathrm{a}_{0}$ configurations, unlike the case of both the EAM potentials predicting metastable states. Furthermore according to the BOP, the repulsive interaction does not vanish with increasing distance in contrast to the ab initio data. The EAM2 potential also predicts remarkable deviation from the $a b$ initio data regarding the binding energy in the range of the interaction distance 0.7$1.1 \mathrm{a}_{0}$. The EAM1 version provides very accurate agreement with the ab initio data not only with respect to the binding energy but also regarding the positions of the $\mathrm{H}$ atoms after the relaxation.

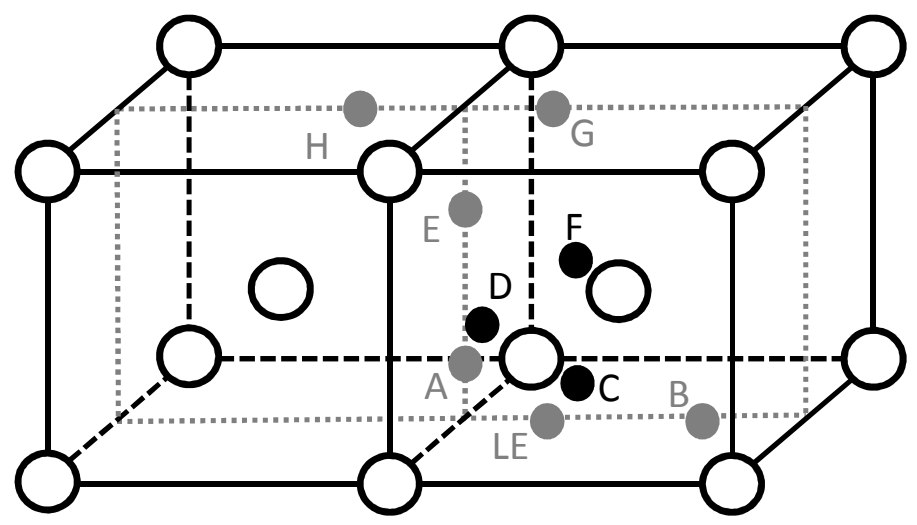

Fig. 1. Schematic picture showing the initial positions used to compute the H-H interaction. Eight pairs of atoms in tetrahedral positions are studied: $\mathrm{H}$ atom marked LE and eight atoms marked in alphabetical order with increasing distance between atoms. Black atoms (D, C, F) lie in the vertical plane facing the picture, gray atoms (LE, A, B, E, H, G) lie in vertical midplane marked by gray dashed lines. Tungsten atoms are presented as empty black circles and form bcc structure.

The binding energy of an interstitial hydrogen to a $\mathrm{H}_{\mathrm{N}}$-vacancy cluster is given in Fig. 3 . We see that the BOP significantly overestimates the attractive interaction for the first, second and third $\mathrm{H}$ atom attached to a single vacancy. In addition, there is a non-monotonic reduction of the binding energy for the fourth and fifth $\mathrm{H}$ atom. The two EAM potentials provide accurate agreement for the binding of $\mathrm{H}_{1}$-vacancy and $\mathrm{H}_{2}$-vacancy complexes and systematically underestimate the biding energy for the larger clusters by about $0.4 \mathrm{eV}$. 


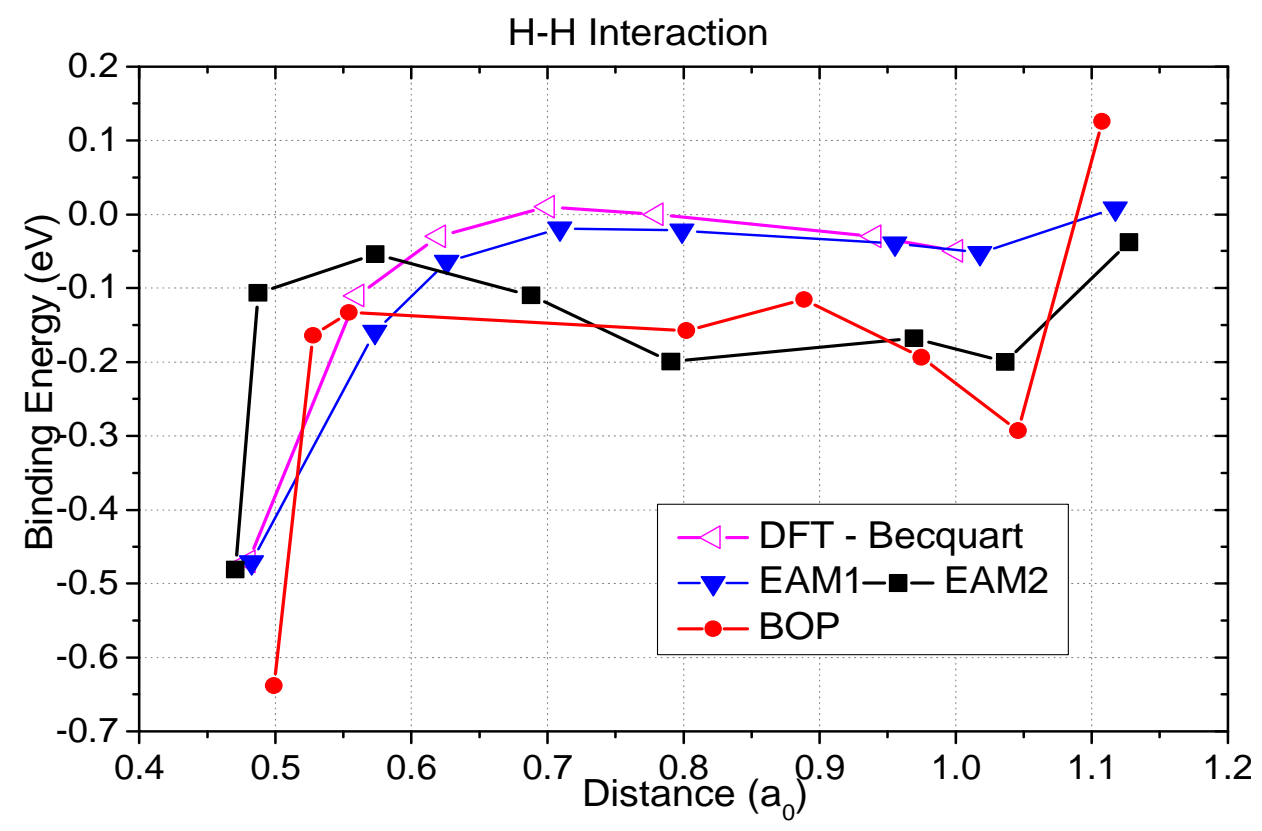

Figure 2. The binding energy for the $\mathrm{H}-\mathrm{H}$ interaction as a function of distance. 'DFT' refers to the ab-initio data taken from [16].

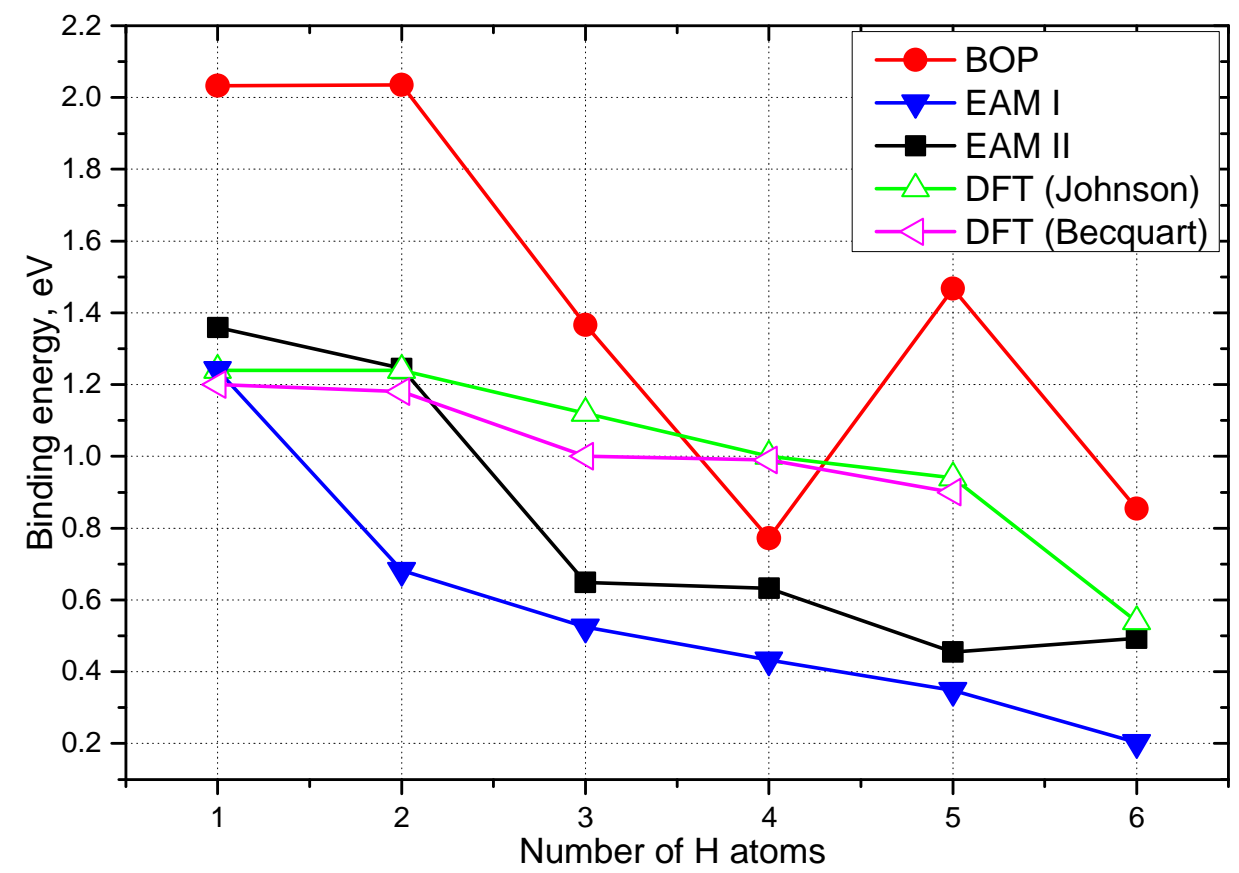

Figure 3. The binding energy for the H-vacancy interaction as a function of distance. 'DFT' refers to the ab-initio data taken from $[16,17]$.

3.2 Interaction of hydrogen with a screw dislocation

Our second set of benchmark calculations consists of the characterization of the interaction of $\mathrm{H}$ with a $1 / 2<111>$ screw dislocation (SD). In our preceding work, we have computed the distribution and corresponding binding energy of $\mathrm{H}$ around the core of the SD. The binding energy map revealed two types of energy minimum configurations for the $\mathrm{H}$ atom: inside the core (three equivalent sites, referred to as 'A' type) and adjacent to the core (six 
equivalent positions, referred to as 'B' type), as is shown in the original work in Fig. 1a [7]. Here, we provide a schematic representation of the location of these positions superposed on the differential displacement maps, calculated using the BOP and EAM potentials, which show the dislocation core structure (see caption of Fig. 4 for a detailed explanation). Note that the BOP potential predicts the three-fold split structure for the dislocation core, which contradicts the $a b$ initio result $[13,18-21]$. Both versions of the EAM potential return the isotropic non-degenerate core structure, which complies with the ab initio data.

The identified positions for a $\mathrm{H}$ atom near the SD core coincide with tetrahedral interstitial sides, which are preferentially occupied by $\mathrm{H}$ atom in bcc $\mathrm{W}$ bulk as well. According to the $a b$ initio results, the binding energy in the two configurations amounts to $0.55 \mathrm{eV}$ and 0.54 $\mathrm{eV}$, i.e., practically being the same. Nor the BOP, neither the EAM potentials could reproduce the $a b$ initio data in full agreement, see Table 1 . While the BOP model predicts reasonable agreement for the binding energy in position $\mathrm{A}$, it overestimates the binding energy by a factor of two in position B. Both versions of the EAM potential do not predict the binding in position A, instead the interaction is practically neutral. The binding energy in the position B is calculated to be $0.42 \mathrm{eV}$ and $0.66 \mathrm{eV}$ for the two versions of the EAM potential, which bounds the ab initio result.
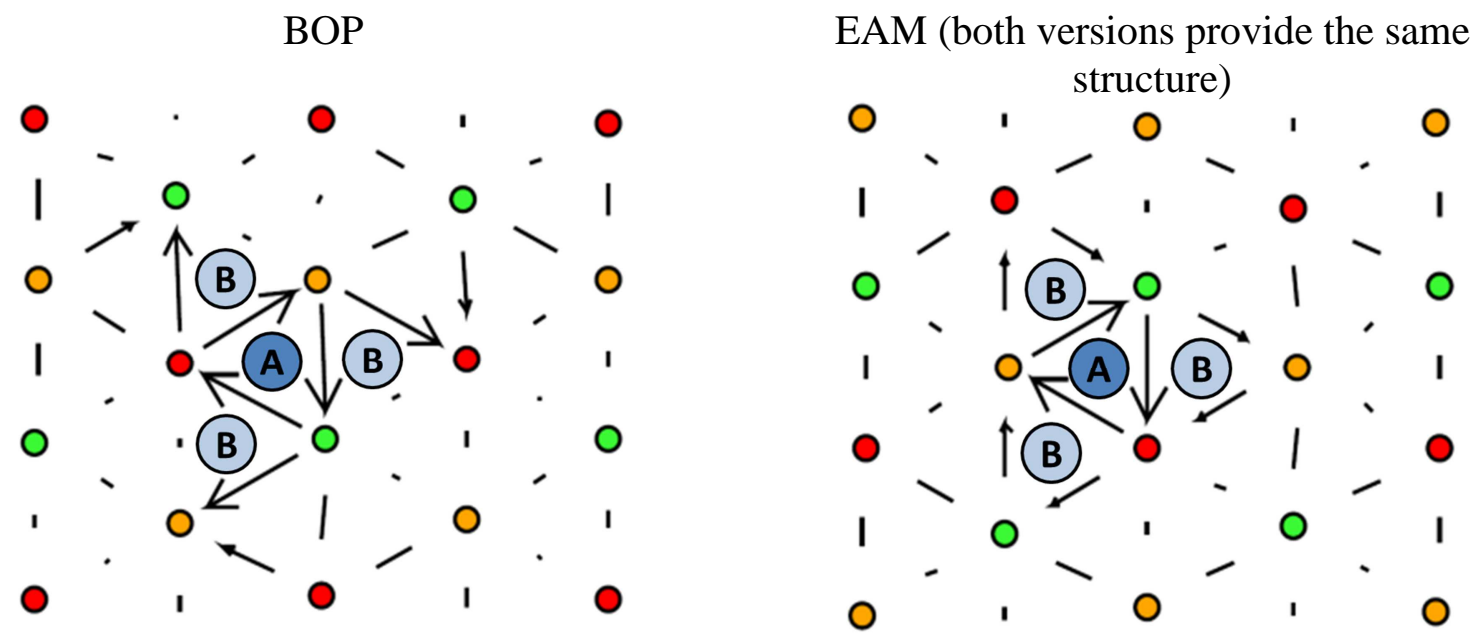

Figure 4. The schematics of the core atoms in a $1 / 2<111\rangle$ screw dislocation in projection onto the (111) plane. The black arrows indicate the difference between displacements of neighboring $<111>$ columns forming the dislocation core. The length of the arrow is proportional to the magnitude of displacement difference, and the direction of the arrow indicates the sign of the displacement difference. Among the three atoms that surround the centre of the dislocation, the arrows form a closed circuit - this is the dislocation core. Note that while the arrows reveal a displacement component in the (111) plane for convenience of visualization, the displacement component they represent is strictly out of the plane. Ground state positions in and next to the dislocation core are schematically shown by light- and dark-blue balls, respectively.

Table 1. Binding energy of H-SD core as predicted according the EAM, BOP and ab-initio method.

\begin{tabular}{|l|l|l|l|l|}
\hline & \multicolumn{3}{l|}{ Binding energy, eV } \\
\hline $\begin{array}{l}\text { Position } \\
\text { type }\end{array}$ & EAM I & EAM II & BOP & $\begin{array}{l}A b \text { initio } \\
{[7]}\end{array}$ \\
\hline A & 0.0 & 0.0 & 0.41 & 0.55 \\
\hline B & 0.42 & 0.66 & 1.03 & 0.54 \\
\hline
\end{tabular}


A description of the edge dislocations using $a b$ initio calculations is computationally heavy and that is probably why there is no ab initio data regarding the interaction of $\mathrm{H}$ with an edge dislocation in bcc W available so far in open source literature. Moreover, we could not find even the $a b$ initio data regarding the properties of edge dislocations in bcc W.

We have constructed a $1 / 2<111>\{110\}$ edge dislocation (ED), as described in Section 2, and relaxed the crystal using the three interatomic potentials. The core structure of the ED was found to be symmetric and extended in the $\{110\}$ glide plane. It was similar with all the applied potentials, see the comparison between the BOP and EAM1 potentials presented in Fig. 5.

The interaction of $\mathrm{H}$ with the core of the ED was studied in all non-equivalent tetra- and octahedral positions above and below the dislocation glide plane. An example of the distribution of the interaction energy is provided in Fig. 6, which was obtained using the EAM2 potential. The binding energy maps calculated using the other potentials were essentially similar. From Fig. 6 it follows that the maximum binding energy is realized if $\mathrm{H}$ is placed in between the two planes forming the imaginary dislocation glide plane. The attractive interaction sharply vanishes as the $\mathrm{H}$ atom is moved above or below the glide plane. While inside the glide plane, the range of the strong interaction is spread over $\sim 10 \AA$, which can be expected given the rather extended structure of the dislocation core (see Fig. 5). The maximum binding energy is found to be 0.63 $\mathrm{eV}, 0.89 \mathrm{eV}$ and $1.64 \mathrm{eV}$ for the EAM1, EAM2 and BOP, respectively. As in the case of the interaction with the SD (in position $\mathrm{B}$ ) and $\mathrm{H}$-vacancy, the BOP predicts a binding energy of a factor two higher than the EAM potentials. Even though we do not have reference ab initio data, we tend to consider that the BOP model overestimates the binding energy following the previous comparisons (see section 3.1 and 3.2).
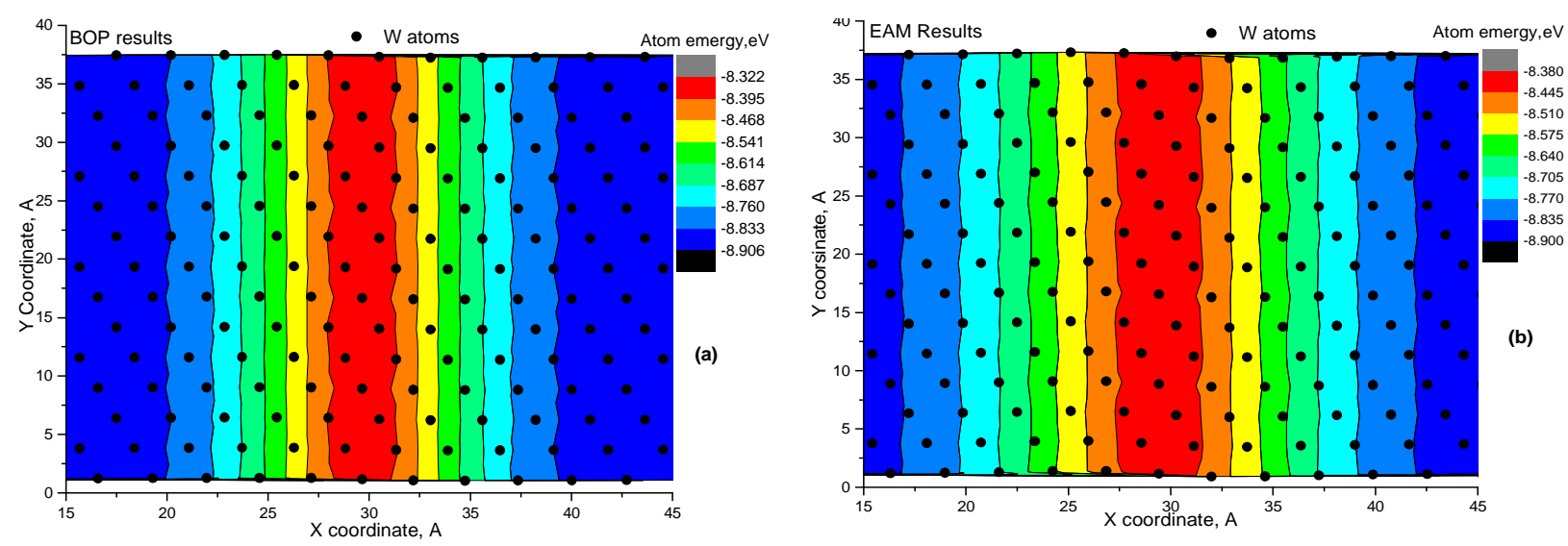

Figure 5. The edge dislocation core structure obtained by the (a) BOP and (b) EAM2 potentials, and shown as the distribution of cohesive energy of the atoms forming the first extra-half $\{110\}$ plane above the imaginary dislocation plane. 


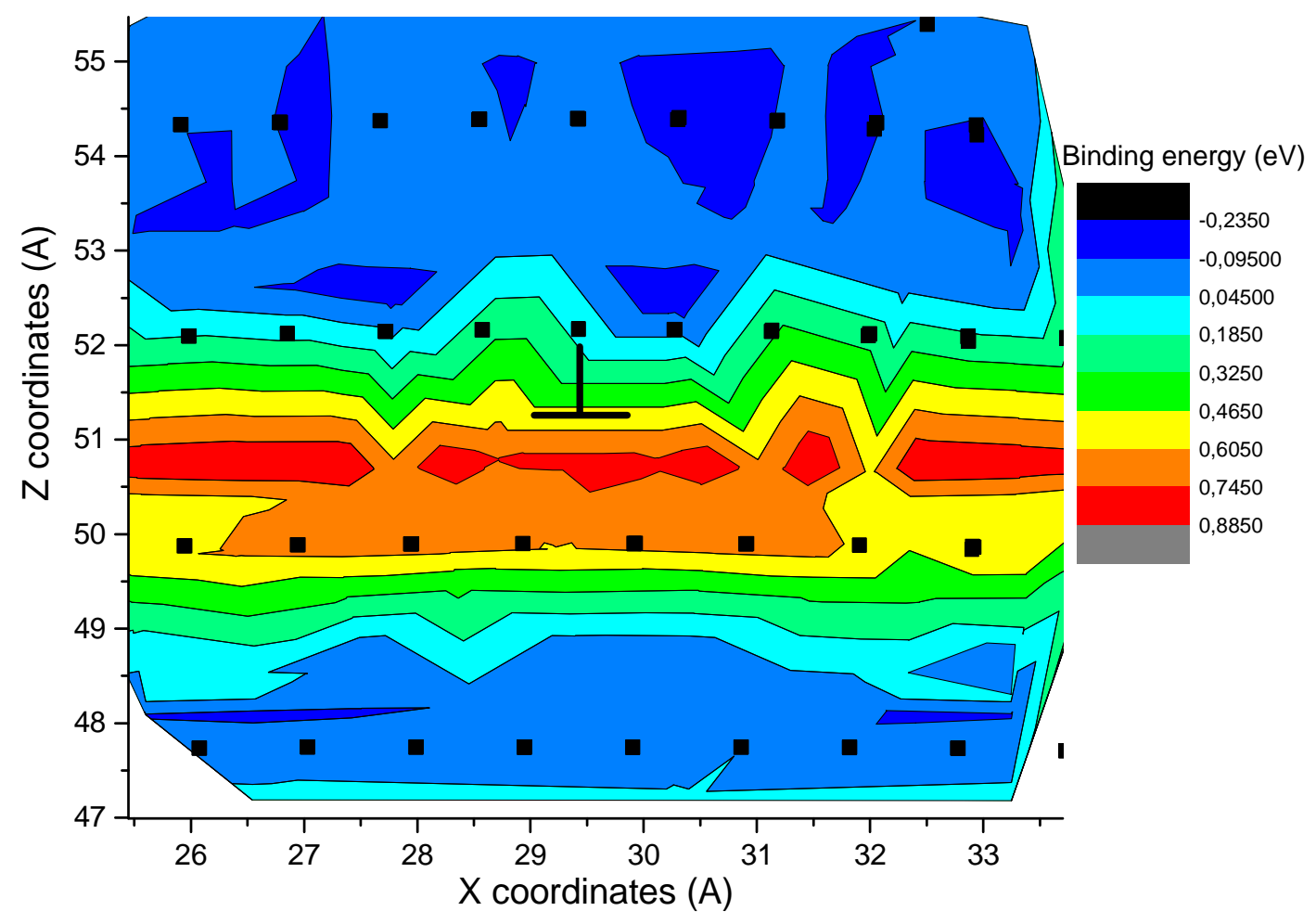

Figure 6. Distribution of the binding energy of $\mathrm{H}$ with the core of the edge dislocation obtained using the EAM2 potential. The geometric center of the dislocation core is shown by the symbol 'L'.

\subsection{Interaction of $\mathrm{H}_{\mathrm{N}}$ clusters with edge dislocation and screw dislocations}

Our next step is to characterize the formation of $\mathrm{H}_{\mathrm{N}}$ clusters on dislocations and deduce the incremental binding energy as a function of cluster size depending on the character of the dislocation. First, we present the result for the SD as this was also studied by $a b$ initio calculations providing us important reference data to judge on the quality of the interatomic potentials.

The incremental binding energy of $\mathrm{H}_{\mathrm{b}}$ to $\mathrm{H}_{\mathrm{N}-1}-\mathrm{SD}$ complex, i.e. binding of an interstitial $\mathrm{H}$ added from the bulk to the $\mathrm{H}_{\mathrm{N}-1}$ cluster placed on the $\mathrm{SD}$, is presented in Fig. 7a. According to the $a b$ initio data, adding the second, third, fourth and fifth $\mathrm{H}$ atom to the cluster progressively reduces the partial binding energy down to $0.35 \mathrm{eV}$. A sudden drop takes place if the seventh $\mathrm{H}$ atom is added, and the recovery for the ninth atom originates from the jog-punching mechanism.

The BOP potential predicts much stronger binding for the second $\mathrm{H}$ atom, while the binding energy for the larger cluster is adequately described up to size $\mathrm{N}=6$. The EAM1 also provides a reasonable description but does not capture the reduction of the binding energy at $\mathrm{N}>$ 6. The EAM2 predicts a flat curve for the binding energy function, as is the case of the EAM1, but overestimates the result by about $0.1-0.2 \mathrm{eV}$ as compared to the DFT data.

The incremental binding energy of a $\mathrm{H}_{\mathrm{SD}}$ to a $\mathrm{H}_{\mathrm{N}-1}-\mathrm{SD}$ complex, i.e. binding of an interstitial $\mathrm{H}$ attached to the SD core with the $\mathrm{H}_{\mathrm{N}-1}$ cluster placed on the $\mathrm{SD}$, is presented in Fig. 7b. Ab initio data suggest that only two $\mathrm{H}$ atoms may form a stable compact complex. Adding more $\mathrm{H}$ atoms should result in the formation of the $\mathrm{H}_{\mathrm{N}}$ clusters 'stretched' along the dislocation line. Both BOP and EAM potentials correctly predict this trend, however, the strength of the interaction differs. The BOP potential provides much larger values for the binding energy, in 
absolute terms, as compared to the both EAM potentials. We can conclude that both types of the potentials predict correctly qualitative trends obtained from the $a b$ initio calculations, while none of the potentials grasp a good quantitative agreement.
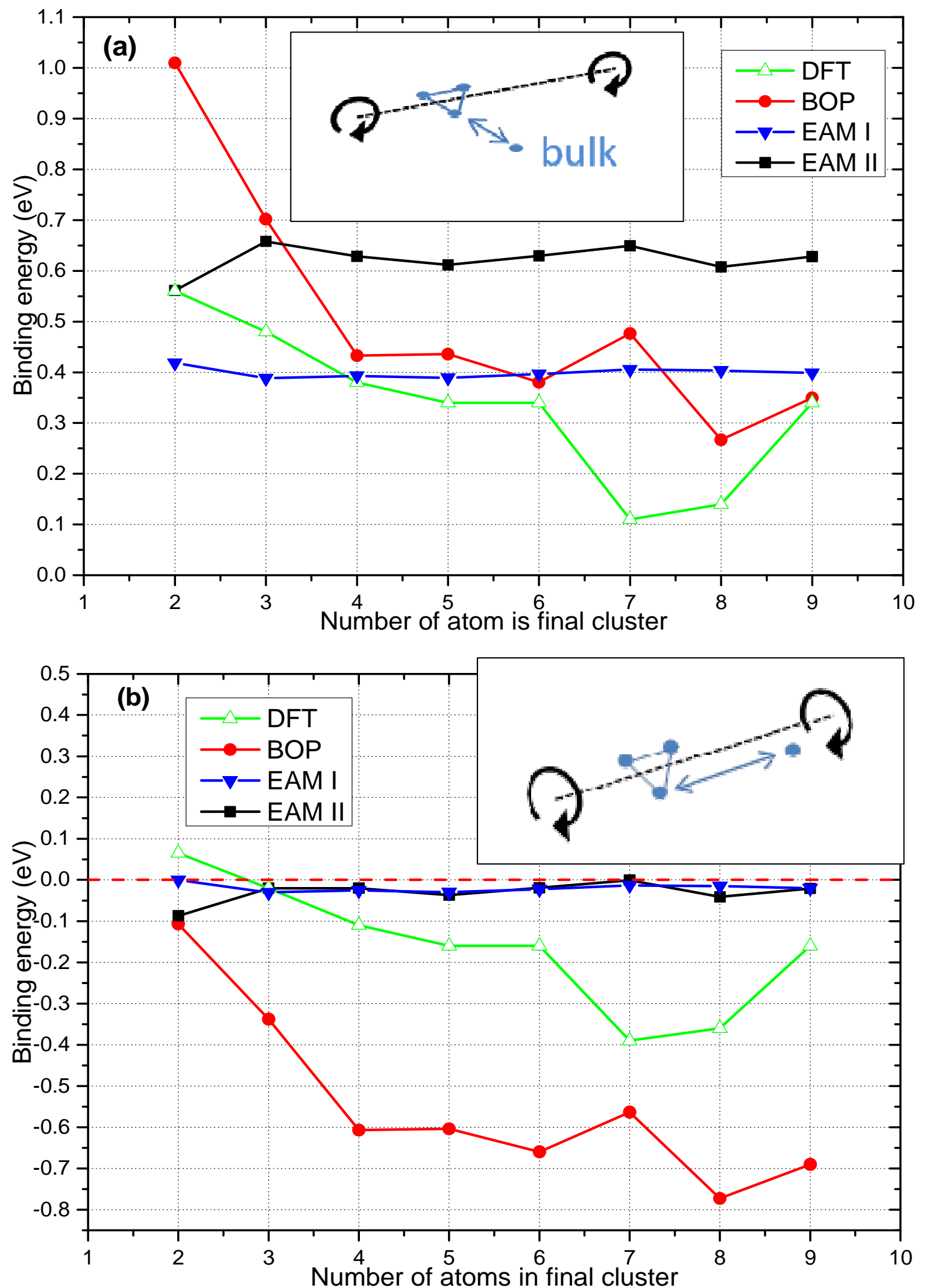

Figure 7. (a) The incremental binding energy of $\mathrm{H}_{\mathrm{b}}$ to $\mathrm{a} \mathrm{H}_{\mathrm{N}-1}$ cluster placed on the SD core. (b) The incremental binding energy of $\mathrm{H}_{\mathrm{SD}}$ to a $\mathrm{H}_{\mathrm{N}-1}$ cluster placed on the $\mathrm{SD}$ core. Inset figures schematically demonstrate the partition of $\mathrm{H}_{\mathrm{b} / \mathrm{SD}}$ and $\mathrm{H}_{\mathrm{N}-1}$. 
The incremental binding energy of $\mathrm{H}_{\mathrm{b}}$ to a $\mathrm{H}_{\mathrm{N}-1}$-ED complex is presented in Fig. 8. It can be seen that all the potentials predict strong attractive interaction of $\mathrm{H}_{\mathrm{b}}$ to the $\mathrm{H}_{\mathrm{N}}$ cluster up to size $\mathrm{N}=5$. The incremental binding energy is of the order of the H-ED binding energy, which implies that $\mathrm{H}$ atoms inside the $\mathrm{H}_{\mathrm{N}}$ cluster can accommodate inside the ED core practically not disturbing each other. This was not the case of the screw dislocation, for which the reduction of the attractive interaction of $\mathrm{H}_{\mathrm{b}}$ to the $\mathrm{H}_{\mathrm{N}-1}-\mathrm{SD}$ was seen already staring from $\mathrm{N}=2$. This result reflects that the space available for the formation of the energetically stable $\mathrm{H}_{\mathrm{N}}$ cluster is essentially larger in the core of the ED as compared to that in the SD core.

The incremental binding energy of a $\mathrm{H}_{\mathrm{ED}}$ to a $\mathrm{H}_{\mathrm{N}-1}$-ED complex is also given in Fig. 8. The data suggest the absence of attractive interaction between $\mathrm{H}$ atoms moving along the ED core. Just as in the case of the screw dislocation, $\mathrm{H}_{\mathrm{N}}$ clusters are expected to grow preferentially forming configurations 'stretched' along the dislocation line. All three interatomic models predict the same trend, but according to the EAM1 the interaction of a $\mathrm{H}_{\mathrm{ED}}$ with a $\mathrm{H}_{\mathrm{N}-1}$-ED cluster is practically neutral.

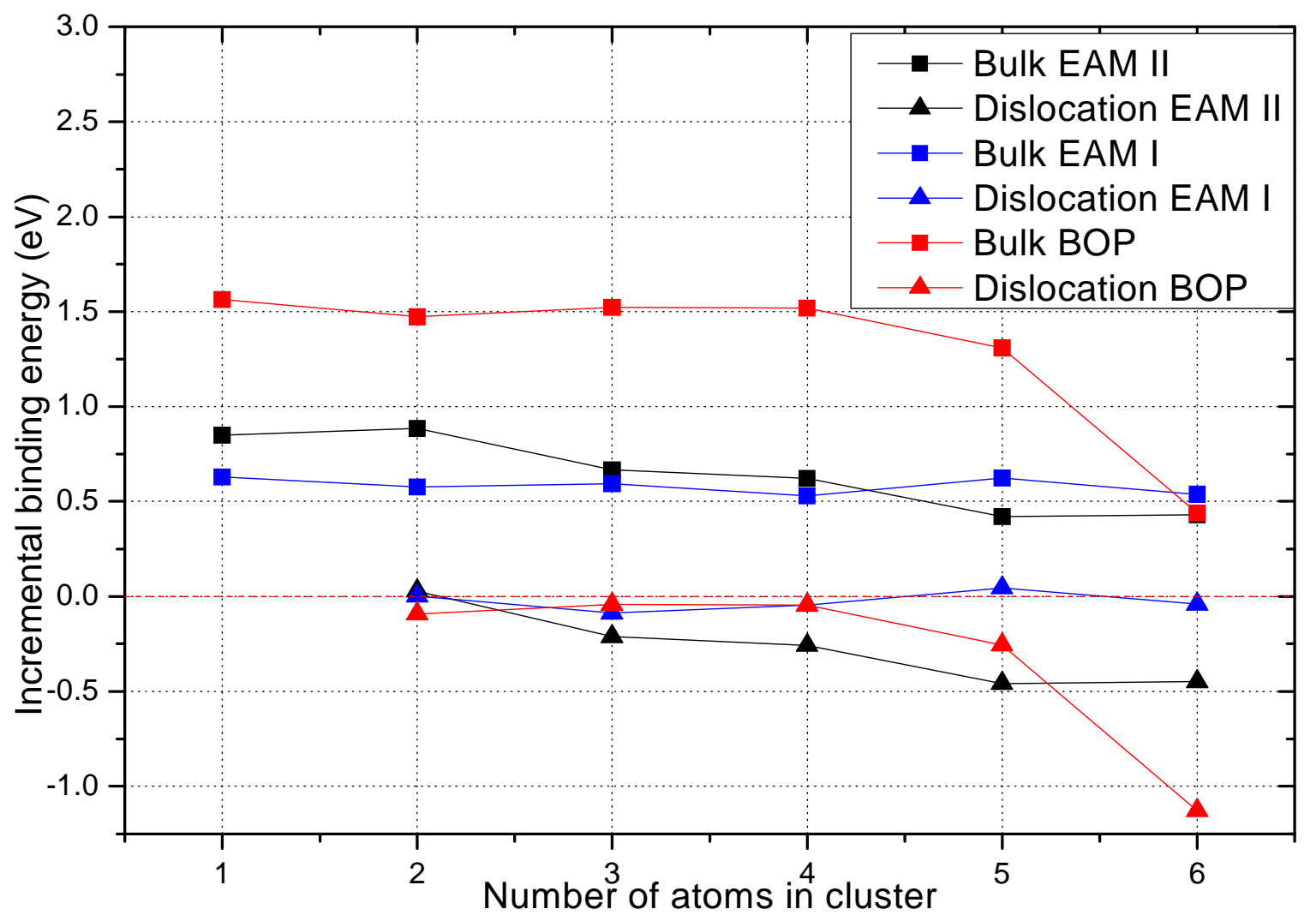

Figure 8. Incremental binding energy of $\mathrm{H}_{\mathrm{b}}-\mathrm{H}_{\mathrm{N}-1}-\mathrm{ED}$ ('Bulk') and $\mathrm{H}_{\mathrm{ED}}-\mathrm{H}_{\mathrm{N}-1}-\mathrm{ED}$ ('Dislocation') obtained by the BOP and EAM potentials.

\section{Diffusion of $\mathrm{H}$ in the dislocation core}

MD simulations to study the diffusion of $\mathrm{H}$ in a crystal containing a ED were done only using the EAM2 potential. Since these calculations were computationally heavy (due to large crystal and a relatively long MD run necessary to achieve satisfactory statistic), we have excluded the BOP potential because of the discrepancies and drawbacks found in the description of the H-defect interaction presented above and also due to the poor performance regarding the properties of dislocations as studied earlier [13]. 
Following and visualizing movement of $\mathrm{H}$ atom we found that it exhibits one dimensional migration along the dislocation core moving by jumping between the planes bounding the imaginary dislocation glide plane. At temperatures below $1300 \mathrm{~K}, \mathrm{H}$ was attached to ED core for the whole time span of the MD run. This behavior is consistent with the strong attractive interaction of a $\mathrm{H}$ to a $\mathrm{ED}\left(\mathrm{E}_{\mathrm{b}}=0.63 / 0.89 \mathrm{eV}\right)$. Above $1300 \mathrm{~K}$, we could regularly register detachment of the $\mathrm{H}$ atom. The trajectory of the $\mathrm{H}$ atom whilst migrating along the dislocation core was therefore reconstructed to obtain the diffusion coefficient from high temperature MD simulations. The resulting diffusion coefficient for 1D-migration along the ED core is drawn in Fig. 9 as a function of temperature. The extracted $D_{0}$ and $E_{m}$ are, respectively, $8.1 \times 10^{-9} \mathrm{~m}^{2} / \mathrm{s}$ and $0.17 \mathrm{eV}$. Note that this value is significantly smaller than the migration energy of a $\mathrm{H}$ in $\mathrm{W}$ bulk, estimated experimentally to be $0.4 \mathrm{eV}$ [22] and obtained by MD: $0.23 \mathrm{eV}$. The experimentally measured [22] and calculated here with the same potential 3D bulk diffusion coefficient is also drawn in Fig. 9 for comparison. The calculated value of $E_{m}$ is $0.23 \mathrm{eV}$ and is lower than the experimentally obtained value, but is consistent with the values of the migration barrier between tetrahedral positions, which is $0.21 \mathrm{eV}$ as predicted by the potential [11], and $0.2 \mathrm{eV}$ as obtained by $a b$ initio in [16]. Clearly, the diffusivity of $\mathrm{H}$ attached to the dislocation line is much higher than the bulk diffusivity, especially at low temperatures as can be seen in Fig. 9.

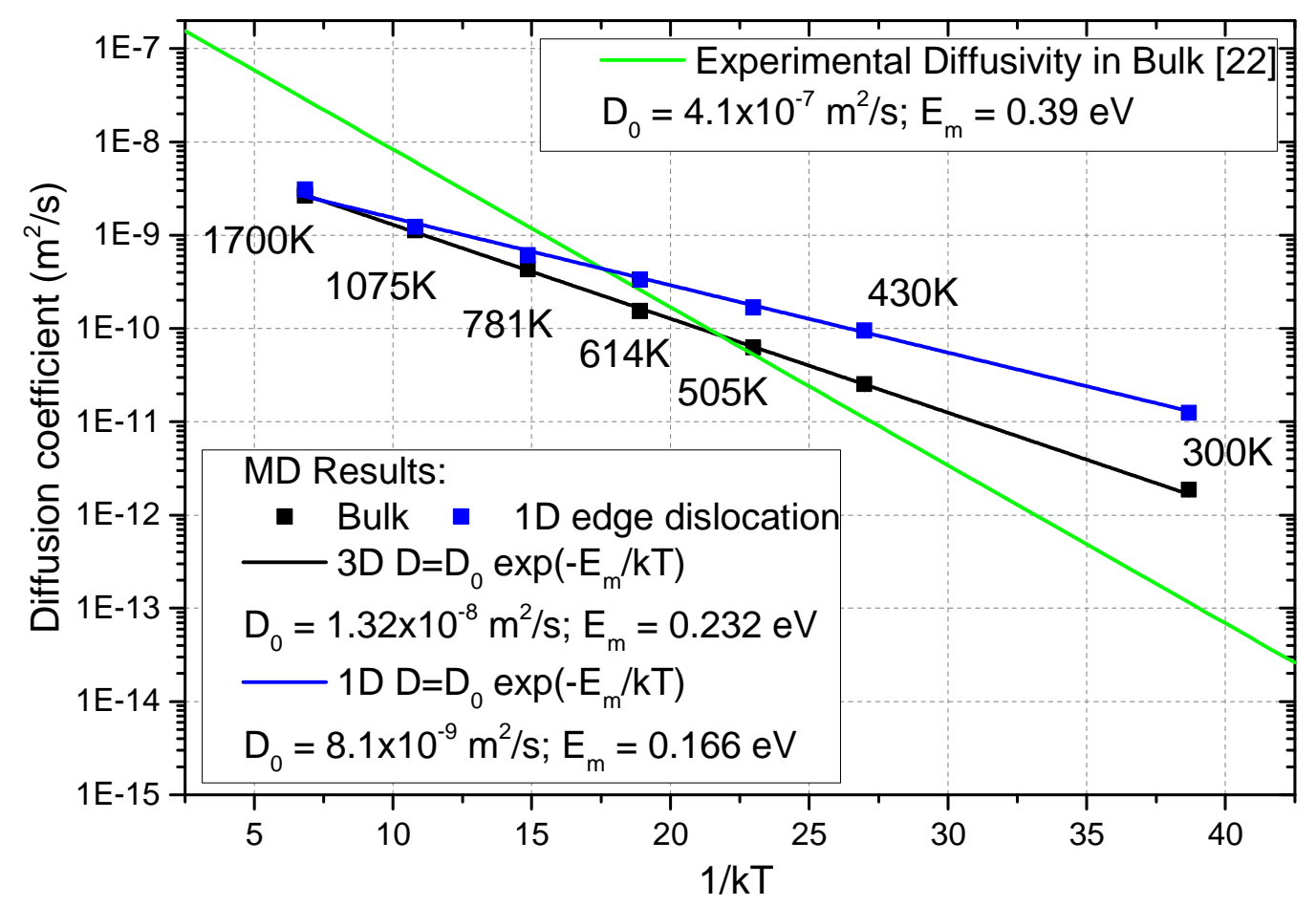

Figure 9. 1-D diffusion coefficient of $\mathrm{H}$ in the core of the edge dislocation and 3D bulk diffusion coefficient as calculated using the EAM2 potential and drawn according to the experimental measurements [22].

\section{Conclusions.}

To summarize, we have performed static and finite temperature simulations to characterize the interaction of $\mathrm{H}$ and hydrogen clusters with different types of dislocations in BCC W. Two types of interatomic potentials were used, namely: the bond order type developed by Li et.al. [9, 10], 
and recently derived embedded atom method potentials by Bonny et al. [11]. On the basis of the obtained results we can draw the following conclusions:

a. By comparing the performance of the two types of potentials regarding the description of $\mathrm{H}$ vacancy and $\mathrm{H}$-dislocation interaction, we conclude that the EAM potential retains a better agreement with $a b$ initio data and therefore was chosen for MD simulations. The BOP model does not provide satisfactory description of the $\mathrm{H}-\mathrm{H}$ interaction embedded in bcc $\mathrm{W}$ bulk, which was important for the present study. In contrast to it, the EAM1 potential provides very good agreement with ab-initio data. Furthermore, the $\mathrm{H}_{\mathrm{N}}$-vacancy interaction is well described by the EAM potential for $\mathrm{N}=1,2$, however, the strength of the binding is systematically underestimated. The BOP overestimates the binding by more than $0.5 \mathrm{eV}$ for $\mathrm{N}=1,2$ and provides non-monotonic reduction of the binding energy for $\mathrm{N}=4,5$ deviating from the trend obtained using ab initio techniques.

b. The results for the interaction of $\mathrm{H}$ with a screw dislocation core reveal that both types of potentials exhibit some disagreements being compared to the $a b$ initio data. The BOP overestimates the interaction in the positions adjacent to the SD core, while the EAM potentials underestimate the interaction in the positions inside the dislocation core. In addition, the BOP fails to reproduce the equilibrium structure of the SD core, and predicts a dissociated three-fold structure in contrast to the ab initio result. The EAM potentials do not have this caveat.

c. The interaction of the $\mathrm{H}$ with the edge dislocation core was not studied by means of ab initio data so far, and therefore we do not have reference data to compare the results obtained with the potentials. However, a qualitative result - strong and localized attraction of $\mathrm{H}$ to the core of the edge dislocation - is independent of the applied potential. Quantitatively, the BOP predicts the binding energy approximately twice as high as compared to both applied EAM potentials. This is overall consistent with the deviation of the results obtained using the BOP and EAM potentials in calculations involving a vacancy and screw dislocation.

d. The analysis of the interaction of $\mathrm{H}_{\mathrm{N}}$ clusters with the edge dislocation reveals a strong tendency to form compact $\mathrm{H}_{\mathrm{N}}$ clusters without losing the binding strength up to five $\mathrm{H}$ atoms. Thus, the core of the edge dislocation can accept twice as much $\mathrm{H}$ atoms as compared to the screw dislocation. This reflects the fact that the space available for the $\mathrm{H}_{\mathrm{N}}$ cluster is essentially larger in the core of the edge dislocation, as one should expect.

e. Based on the MD data obtained here using the EAM2 model, we conclude that $\mathrm{H}$ exhibits strong attractive interaction to the core of the $1 / 2<111>\{110\}$ edge dislocation, consistent with MS results, and performs one-dimensional migration, which is remarkably faster as compared to the bulk diffusivity.

The last conclusion implies that not only a single $\mathrm{H}$ but also multiple energetically stable $\mathrm{H}_{\mathrm{N}}$ clusters may exhibit significant diffusivity along the dislocation core. Investigation of the dynamical behavior of $\mathrm{H}_{\mathrm{N}}$ clusters and possible mechanisms leading to the 'jog-punching' on the edge dislocation is currently ongoing. 
Acknowledgements:

This work was supported by the European Commission and carried out within the framework of the Erasmus Mundus International Doctoral College in Fusion Science and Engineering (FUSION-DC). The work is partially supported by EUROfusion program.

References:

1. R.E. Clark and D. Reiter, Nuclear fusion research: understanding plasma-surface interactions, Springer, 2005.

2. Y. Zayachuk, M.H.J.t. Hoen, P.A.Z.v. Emmichoven, I. Uytdenhouwen, and G.v. Oost, Nucl. Fusion 52 (2012) 103021.

3. O.V. Ogorodnikova, J. Roth, and M. Mayer, J. Nucl. Mater. 313-316 (2003) 469.

4. V.K. Alimov, B. Tyburska-Püschel, S. Lindig, Y. Hatano, M. Balden, J. Roth, et al., J. Nucl. Mater. 420 (2012) 519.

5. $\quad$ T. Ahlgren, K. Heinola, K. Vörtler, and J. Keinonen, J. Nucl. Mater. 427 (2012) 152.

6. A.A. Haasz, J.W. Davis, M. Poon, and R.G. Macaulay-Newcombe, J. Nucl. Mater. 258-263, Part 1 (1998) 889.

7. D. Terentyev, V. Dubinko, A. Bakaev, Y. Zayachuk, W.V. Renterghem, and P. Grigorev, Nucl. Fusion 54 (2014) 042004.

8. V.I. Dubinko, P. Grigorev, A. Bakaev, D. Terentyev, G. van Oost, F. Gao, et al., J. Phys.: Condens. Matter. 26 (2014) 395001.

9. X.-C. Li, X. Shu, Y.-N. Liu, Y. Yu, F. Gao, and G.-H. Lu, J. Nucl. Mater. 426 (2012) 31.

10. X.-C. Li, X. Shu, Y.-N. Liu, F. Gao, and G.-H. Lu, J. Nucl. Mater. 408 (2011) 12.

11. G. Bonny, P. Grigorev, and D. Terentyev, J. Phys.: Condens. Matter. 26 (2014) 485001.

12. M.-C. Marinica, L. Ventelon, M.R. Gilbert, L. Proville, S.L. Dudarev, J. Marian, et al., J. Phys.: Condens. Matter. 25 (2013) 395502.

13. G. Bonny, D. Terentyev, A. Bakaev, P. Grigorev, and D.V. Neck, Modelling and Simulation in Materials Science and Engineering 22 (2014) 053001.

14. K. Heinola, T. Ahlgren, K. Nordlund, and J. Keinonen, Phys. Rev. B 82 (2010) 094102.

15. S. Plimpton, J. Comput. Phys. 117 (1995) 1.

16. C.S. Becquart and C. Domain, J. Nucl. Mater. 386-388 (2009) 109.

17. D.F. Johnson and E.A. Carter, J. Mater. Res. 25 (2010) 315.

18. L. Romaner, C. Ambrosch-Draxl, and R. Pippan, Phys. Rev. Lett. 104 (2010) 195503.

19. H. Li, S. Wurster, C. Motz, L. Romaner, C. Ambrosch-Draxl, and R. Pippan, Acta Materialia 60 (2012) 748.

20. G.D. Samolyuk, Y.N. Osetsky, and R.E. Stoller, J. Phys.: Condens. Matter. 25 (2013) 025403.

21. S.L. Frederiksen and K.W. Jacobsen, Philos. Mag. 83 (2003) 365.

22. R. Frauenfelder, J. Vac. Sci. Technol. 6 (1969) 388. 